\title{
Las asociaciones de ostrácodos en secuencias aluviales como indicadores de cambios ambientales holocenos (Bardenas Reales de Navarra, Cuenca del Ebro, NE Península Ibérica)
}

\author{
The ostracod assemblages in alluvial sequences as indicators \\ of Holocene environmental changes (Bardenas Reales de \\ Navarra, Ebro Basin, NE Iberian Peninsula)
}

\author{
B. Martínez-García ${ }^{1}$, O. Suarez-Hernando ${ }^{1}$, J. Rodríguez-Lázaro ${ }^{1}$, A. Pascuall ${ }^{1}$, A. Ordiales ${ }^{1}$, \\ X. Murelaga ${ }^{1}$, C. Sancho ${ }^{2}$, A. Muñoz ${ }^{2}$, C. Osácar ${ }^{2}$ \\ 1 Departamento de Estratigrafía y Paleontología, Universidad del País Vasco UPV/EHU, 48080 Bilbao, España. \\ Email: blancamaria.martinez@ehu.eus \\ 2 Departamento de Ciencias de la Tierra, Universidad de Zaragoza, 50009 Zaragoza, España
}

\section{RESUMEN}

\begin{abstract}
En este trabajo se estudian las asociaciones de ostrácodos identificadas en tres unidades aluviales preservadas en Bardenas Reales de Navarra (Cuenca del Ebro, NE Península Ibérica), para reconstruir la evolución paleoambiental de esta área durante el Holoceno final. Las especies de ostrácodos más comunes en las muestras estudiadas son Ilyocypris bradyi Sars y Paralimnocythere aff. psammophila (Flössner), acompañadas por Pseudocandona albicans (Brady). Las variaciones en su abundancia relativa a lo largo de dichas unidades aluviales permiten caracterizar cambios internos en el ambiente de depósito. Así, la unidad depositada entre $4763 \pm 87$ y $2848 \pm 55$ años cal. BP presenta como especie más abundante a $/$. bradyi, lo que indica la existencia de pequeños arroyos o riachuelos con agua corriente que se desarrollaron probablemente en llanuras de inundación fluviales. Sin embargo, de $2848 \pm 55$ a $836 \pm 65$ años cal. BP Paralimnocythere aff. psammophila es la especie dominante, representando el establecimiento de un medio acuático efímero y de aguas estancadas, posiblemente relacionado con condiciones climáticas más áridas (Anomalía Climática Medieval). Finalmente, la asociación de ostrácodos

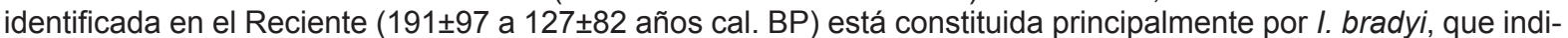
caría la presencia de nuevo de aguas corrientes. Esta última asociación parece estar vinculada a la influencia de condiciones climáticas húmedas y frías (Pequeña Edad del Hielo) durante el Reciente en esta área. Por lo tanto, la evolución paleoambiental para el Holoceno final de Bardenas Reales de Navarra, identificada a partir de las asociaciones de ostrácodos, muestra una buena correlación con los cambios climáticos rápidos holocenos deducidos en otros registros continentales del NE de la Península Ibérica.
\end{abstract}

Palabras clave: Ostrácodos; Registros aluviales; Cambios ambientales; Holoceno final; Bardenas Reales de Navarra.

Recibido el 19 de febrero de 2015 / Aceptado el 30 de septiembre de 2015 / Publicado online el 20 de noviembre de 2015

Citation / Cómo citar este artículo: B. Martínez-García, et al. (2015). Las asociaciones de ostrácodos en secuencias aluviales como indicadores de cambios ambientales holocenos (Bardenas Reales de Navarra, Cuenca del Ebro, NE Península Ibérica). Estudios Geológicos 71(2): e042. http://dx.doi.org/10.3989/egeol.42054.367.

Copyright: () 2015 CSIC. This is an open-access article distributed under the terms of the Creative Commons Attribution-Non Commercial (by-nc) Spain 3.0 License. 


\section{ABSTRACT}

In this paper, the ostracod assemblages identified in three alluvial units preserved in Bardenas Reales de Navarra (Ebro Basin, NE Iberian Peninsula) have been studied, with the aim of reconstructing the palaeoenvironmental evolution of this area during the late Holocene. The most common ostracod species in the studied samples are Ilyocypris bradyi Sars and Paralimnocythere aff. psammophila (Flössner), accompanied by Pseudocandona albicans (Brady). Variations in their relative abundance along these alluvial units allow us to characterize internal changes in the depositional environment. Thus, the unit deposited between $4763 \pm 87$ and $2848 \pm 55$ years cal. BP is dominated by 1 . bradyi, indicating the presence of small streams with running water developed probably in fluvial flood plains. Nevertheless, from $2848 \pm 55$ to $836 \pm 65$ years cal. BP Paralimnocythere aff. psammophila is the dominant species, representing the establishment of an ephemeral and stagnant aquatic environment, mainly related with more arid climatic conditions (Medieval Warm Period). Finally, the ostracod assemblage identified in Recent times $(191 \pm 97$ to $127 \pm 82$ years cal. BP) is mainly dominated by $I$. bradyi, which indicates the presence of running waters. This later assemblage probably defines the influence of cold and wet climatic phase (Little Ice Age) during Recent times in this area. The palaeoenvironmental evolution identified from the late Holocene according to ostracod assemblages in Bardenas Reales de Navarra shows good correlation with rapid climatic changes deduced from other continental records of the NE Iberian Peninsula.

Keywords: Ostracods; Alluvial sequences; Environmental changes; late Holocene; Bardenas Reales de Navarra.

\section{Introducción}

Diversos trabajos han constatado la importancia de los registros aluviales como indicadores de cambios ambientales en áreas semiáridas de todo el mundo (e.g. Chorley et al., 1984; Knox, 1984; Bull, 1991; Peña et al., 2000; González, 2001; Faust et al., 2004). Esto es debido a que los sistemas aluviales semiáridos son muy sensibles a los cambios climáticos de alta frecuencia, tales como los ocurridos durante el Holoceno (Trudgill, 1976; Chorley et al., 1984; Viles \& Goudie, 2003; Faust et al., 2004; entre otros), así como a eventos extremos de alta magnitud (Eybergen \& Imeson, 1989; González, 2001).

Además de indicadores sedimentológicos, mineralógicos y geoquímicos, los registros aluviales incluyen otros de naturaleza biológica. En ocasiones han sido utilizados gasterópodos, fitolitos y biominerales. Estos archivos aluviales también contienen ostrácodos, si bien han sido objeto de escasa atención.

Los ostrácodos, que son un grupo de microcrustáceos con un caparazón bivalvado de composición calcítica fácilmente preservable en el registro fósil (e.g. Rodríguez-Lázaro \& Ruiz-Muñoz, 2012), se han evidenciado como una importante herramienta en las reconstrucciones paleoambientales, debido a su rápida respuesta ante cambios en el medio que habitan.

Estos microorganismos viven en todo tipo de ambientes acuáticos, desde continentales hasta marino profundos y desde agua dulce hasta agua hipersalina (Horne et al., 2002; Rodríguez-Lázaro \& Ruiz-Muñoz, 2012). Su distribución en el medio está relacionada con diversos parámetros ambientales, como la salinidad, la temperatura, la profundidad, el $\mathrm{pH}$, el oxígeno disuelto, el tipo de sedimento o las fuentes de alimento (Meisch, 2000; Rodríguez-Lázaro \& Ruiz-Muñoz, 2012; entre otros). Así mismo, en función del medio que habitan, pueden presentar diferentes comportamientos, siendo planctónicos, bentónicos, nectónicos o endobentónicos (e.g. Meisch, 2000).

Esta versatilidad ha suscitado su empleo en numerosos trabajos sobre estudios ambientales en medios continentales europeos, principalmente lacustres, tanto actuales como holocenos (e.g. De Deckker \& Forester, 1988; Griffiths \& Holmes, 2000; Meisch, 2000; Boomer et al., 2003; Mezquita et al., 2005; Reed et al., 2012). Sin embargo, hay que tener en cuenta que, en medios continentales y más especialmente en ambientes fluviales o aluviales, sin cuerpos de aguas estables, las poblaciones de ostrácodos aparecen de forma discontinua. En áreas de fuerte hidrodinamismo o en zonas de escorrentía, además, el grado de conservación es muy deficiente, por lo que la presencia de ostrácodos es rara (Poquet \& Mesquita-Joanes, 2011).

Existen antecedentes sobre estudios morfoestratigráficos, sedimentológicos y cronológicos detallados de las secuencias aluviales holocenas preservadas en Bardenas Reales de Navarra (Sancho et al., 2008; Murelaga et al., 2012; Bastida et al., 2013; GómezPaccard et al., 2013). Así mismo, han sido analizadas las asociaciones de gasterópodos presentes en estos sedimentos, efectuando además análisis isotópicos de sus conchas (Murelaga et al., 2008; Ortega et al., 2009; Murelaga et al., 2012), aportando una valiosa información paleoambiental durante el Holoceno final a escala regional en esta área. 
A pesar de que los ostrácodos están presentes en estos sedimentos aluviales, no se habían realizado estudios paleoambientales previos empleando estos microcrustáceos. Por tanto, en este trabajo se analizan por vez primera las asociaciones específicas de ostrácodos identificadas en los sedimentos aluviales holocenos de Bardenas Reales de Navarra, con el objetivo de complementar las reconstrucciones paleoambientales anteriormente realizadas.

\section{Área de estudio}

Bardenas Reales de Navarra es un Parque Natural, Lugar de Interés Comunitario y Reserva de la Biosfera localizado al noreste de la Península Ibérica, concretamente al sureste de la Comunidad Foral de Navarra (Fig. 1). Presenta un clima semiárido de fuertes contrastes climáticos, con temperaturas medias de unos $6{ }^{\circ} \mathrm{C}$ en invierno y $24{ }^{\circ} \mathrm{C}$ en verano y una precipitación media variable a lo largo del año, caracterizada por cortos pero intensos periodos de lluvia durante primavera y otoño.

La parte central de Bardenas Reales está formada por una extensa depresión semi-cerrada, denominada Bardena Blanca (Fig. 1). Esta depresión está rodeada en su margen $\mathrm{N}$ por altos cabezos (e.g. La Estroza o Cornialto) cubiertos por sedimentos aluviales del Pleistoceno inferior. En el margen S, la depresión de la Bardena Blanca está flanqueada por relieves estructurales (e.g. Pilatos o Tripa Azul) formados

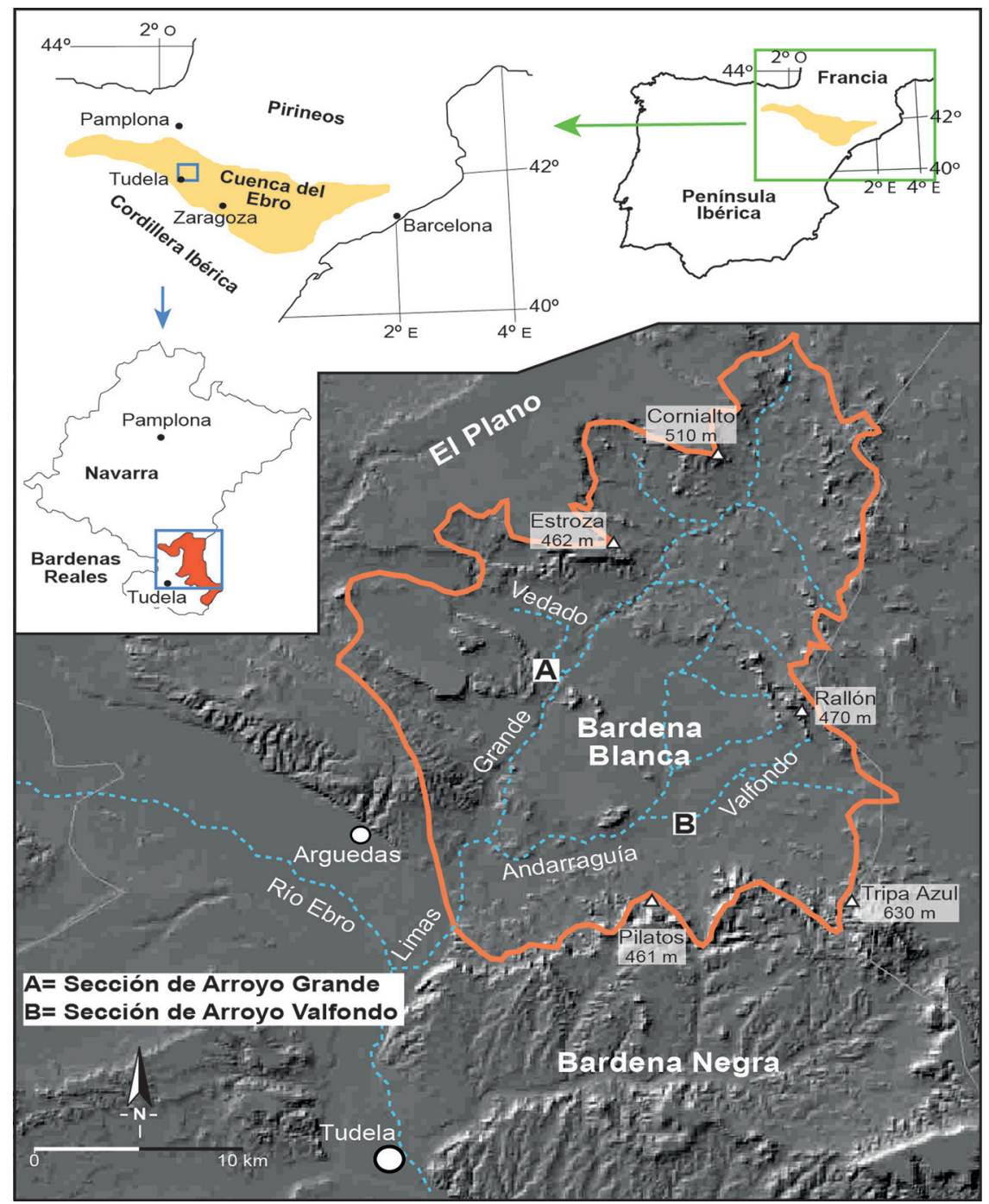

Fig. 1.-Localización geográfica del área de estudio (modificado de Murelaga et al., 2012). 
por sedimentos del Oligoceno y Mioceno (principalmente lutitas, calizas y areniscas; Larrasoaña et al., 2006) de la Bardena Negra.

El fondo de esta depresión (Bardena Blanca) se encuentra tapizado por potentes secuencias aluviales holocenas de gran continuidad. Se diferencian cuatro unidades morfosedimentarias aluviales desarrolladas en dos estadios paleogeográficos (Sancho et al., 2008). El primer estadio (Holoceno inferior) corresponde a una depresión casi cerrada que incluye una primera Unidad Morfosedimentaria (UM 1), de gran extensión, formada por una secuencia compleja de agradación, en la que se diferencian un sistema fluvial anastomosado, una llanura de inundación limo-arenosa y un complejo sedimentario de lagoplaya (Sancho et al., 2008). El segundo estadio paleogeográfico corresponde al exorreismo completo de la depresión anterior, donde la actividad aluvial está relacionada con la red de drenaje actual (sistema de los arroyos Limas-Grande-Andarraguía; ver Fig. 1), de manera que es posible diferenciar tres Unidades Morfosedimentarias (UM 2, 3 y 4) que conforman una secuencia de terrazas encajadas de erosiónrelleno pertenecientes al Holoceno medio y superior. La UM 2 está formada por secuencias fluviales con paleosuelos que representan un período de alta inestabilidad geomorfológica, con fases alternantes de incisión de canales y sedimentación aluvial, depositadas sobre una superficie de erosión irregular que afecta tanto a los materiales pleistocenos y holocenos previos como al sustrato mioceno (Sancho et al., 2008). La UM 3, por su parte, representa el depósito de sedimentos en una extensa llanura aluvial desarrollada sobre los materiales previos, que muestra una ligera incisión con respecto a la superficie de la UM 2 y variaciones en su espesor a lo largo de la Bardena Blanca. Finalmente, la UM 4 incluye numerosas secuencias de relleno de canal y de desbordamiento, apareciendo en pequeños afloramientos fuertemente relacionados con la red de drenaje actual de los arroyos de la Bardena Blanca (Sancho et al., 2008).

\section{Metodología}

\section{Secciones estudiadas}

En este trabajo se estudian dos secciones, la de Arroyo Grande (Figs. 1 y 2A) y la de Arroyo Valfondo (Figs. 1 y 2B), descritas por Murelaga et al. (2012), en las que se identifican las unidades morfosedimentarias del Holoceno final (UM 2, 3 y 4) definidas por Sancho et al. (2008). La unidad UM 2 está formada por arenas y limos (Fig. 2), con intercalaciones esporádicas de conglomerados en la sección de Arroyo Grande (Murelaga et al., 2012). Esta unidad se depositó cubriendo una superficie de erosión irregular desarrollada sobre los materiales previos, y puede ser interpretada como un depósito fluvial en una secuencia de incisión y relleno de canales. La unidad UM 3 está constituida por arenas con yesos, alternantes con lutitas y limos laminados, que representan secuencias de incisión y relleno de canales con intercalaciones de depósitos de sedimentos finos posiblemente transportados por eventos esporádicos de lluvias torrenciales (Murelaga et al., 2012). Finalmente, la UM 4 aparece en pequeños afloramientos, estando fuertemente relacionada con los procesos actuales de erosión de barrancos y el relleno de canales incisos previos. En la sección de Arroyo Grande está compuesta por arenas intercaladas con limos (Fig. 2A), mientras que en la sección de Arroyo Valfondo está conformada por limos masivos y laminados (Fig. 2B) (Murelaga et al., 2012). Esta unidad representa el depósito de materiales transportados por paleoinundaciones.

La edad de estas secciones fue estimada por Murelaga et al. (2012) y está basada en nueve dataciones absolutas con ${ }^{14} \mathrm{C}$ realizadas en restos de carbón (Fig. 2; Tabla 1; ver Murelaga et al., 2012 para detalles sobre el procesado de las muestras y la calibración de las edades obtenidas). De acuerdo a estas dataciones, las muestras analizadas abarcarían el intervalo temporal comprendido entre $4763 \pm 87$ años cal. BP y $127 \pm 82$ años cal. BP (Fig. 2; Tabla 1), es decir, desde el final del Holoceno medio hasta el Reciente.

\section{Análisis microfaunístico}

En este trabajo se han estudiado 41 muestras: 30 de ellas obtenidas de la sección de Arroyo Grande (Fig. 2A) y las 11 restantes en la sección de Arroyo Valfondo (Fig. 2B).

En cada nivel seleccionado se han recogido aproximadamente 500 gramos de sedimento, con el objetivo de disponer de un número de ejemplares de ostrácodos que sea representativo de las asociaciones preservadas, dada la pobreza microfaunística del sedimento. Este sedimento ha sido lavado y tamizado, 
(A)

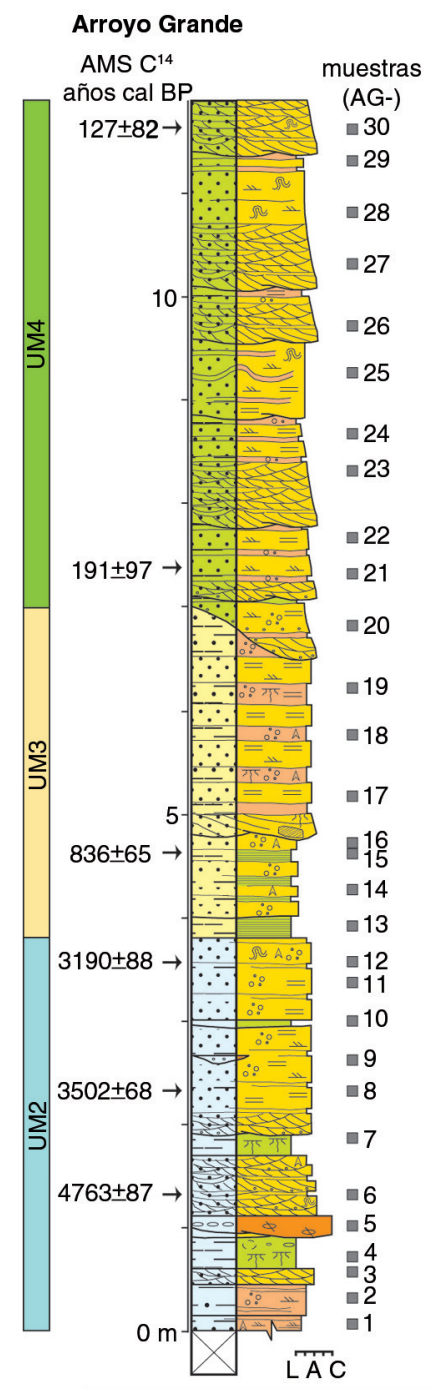

(B)

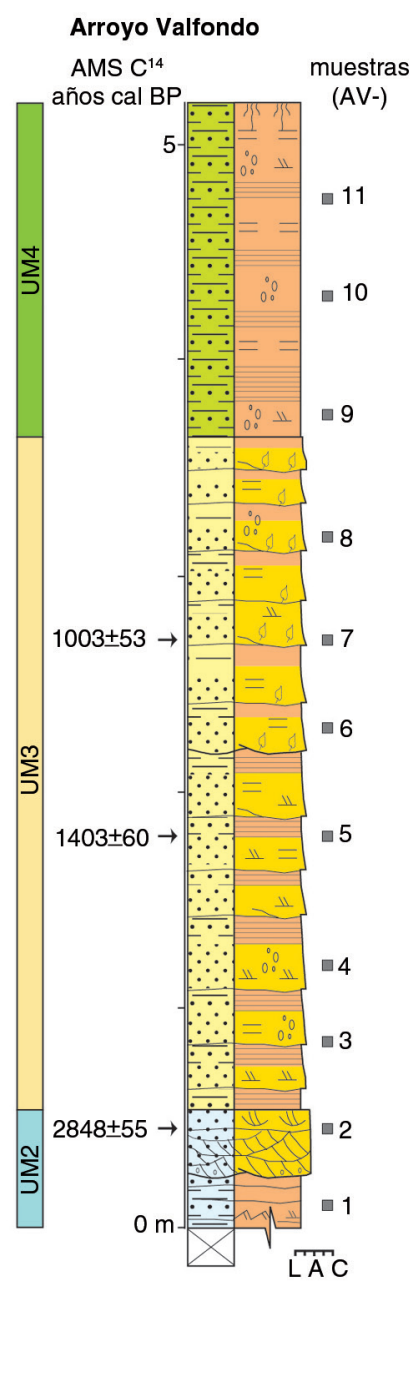

(C)
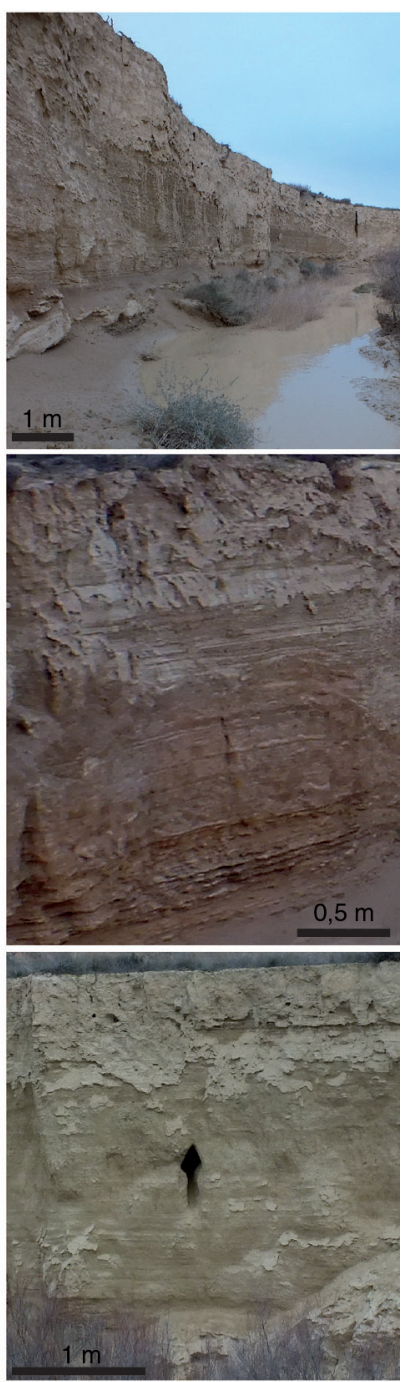

Leyenda:

\begin{tabular}{|c|c|c|c|c|c|c|c|c|c|c|}
\hline Sc & conglome & idos & $\because \because \because$ arena & & $\ldots$ limos & $E=$ Iutitas & \multicolumn{4}{|c|}{$\mathrm{L}=\operatorname{limo} ; \mathrm{A}=$ arena $; \mathrm{C}=$ conglomerado } \\
\hline 凤 & $\begin{array}{l}\text { laminación } \\
\text { convoluta }\end{array}$ & $\phi$ & $\begin{array}{l}\text { cantos } \\
\text { blandos }\end{array}$ & $\therefore$ & vesículas & 雨 bioturbación & $\therefore$ & nódulos & & $\begin{array}{l}\text { laminación } \\
\text { paralela }\end{array}$ \\
\hline 0 & $\begin{array}{l}\text { restos } \\
\text { vegetales }\end{array}$ & 11 & $\begin{array}{l}\text { laminación } \\
\text { cruzada }\end{array}$ & $=$ & carbón & « ripples & $A$ & yeso & $\mathbb{U}$ & $\begin{array}{l}\text { estratificación } \\
\text { cruzada }\end{array}$ \\
\hline & \multicolumn{3}{|c|}{ Unidad Morfosedimentaria 2} & \multicolumn{4}{|c|}{ Unidad Morfosedimentaria 3} & \multicolumn{3}{|c|}{ Unidad Morfosedimentaria 4} \\
\hline
\end{tabular}

Fig. 2.-Perfiles estratigráficos de las secciones de Arroyo Grande (A) y Arroyo Valfondo (B), en los que se detallan las unidades morfológicas identificadas, las características sedimentarias, las edades calibradas y las muestras analizadas. C) Aspecto de campo de los materiales estudiados de la sección de Arroyo Grande: fotografía superior, tramo de 0 a 7 m de la serie (UM 2 y UM 3 ); fotografía central, tramo de 4,8 a 7 m (UM 3); fotografía inferior, tramo de 8 a 11 m (UM 4). Las fotografías no presentan exageración vertical. A) y B), imágenes modificadas de Murelaga et al. (2012).

analizándose la fracción comprendida entre $150 \mu \mathrm{m}$ y $1 \mathrm{~mm}$ de diámetro, de la que se ha extraído la totalidad de los individuos adultos y del último estadio juvenil (A-1) presentes en cada muestra. La fracción superior a $1 \mathrm{~mm}$ no se ha considerado ya que no contenía ejemplares clasificables de ostrácodos. Tanto las valvas desarticuladas como los ejemplares con caparazón completo se consideran como un único individuo. 
Tabla 1.-Dataciones absolutas de radiocarbono (tomadas de Murelaga et al., 2012)

\begin{tabular}{lcccccc}
\hline código del laboratorio & $\begin{array}{c}\text { Unidad Morfo- } \\
\text { sedimentaria }\end{array}$ & muestra & material & localización & $\begin{array}{c}\text { edad } \\
\text { (años BP) }\end{array}$ & $\begin{array}{c}\text { edad calibrada } \\
\text { (años cal. BP) }\end{array}$ \\
\hline UZ5522/ETH34406 & UM 4 & AG-30 & Carbón & Arroyo Grande & $85 \pm 45$ & $127 \pm 82$ \\
UZ5521/ETH34405 & UM 4 & AG-21 & Carbón & Arroyo Grande & $210 \pm 45$ & $191 \pm 97$ \\
UZ5627/ETH35393 & UM 3 & AG-15 & Carbón & Arroyo Grande & $925 \pm 65$ & $836 \pm 65$ \\
UZ5524/ETH34408 & UM 3 & AV-7 & Carbón & Arroyo Valfondo & $1085 \pm 50$ & $1003 \pm 53$ \\
UZ5631/ETH35396 & UM 3 & AV-5 & Carbón & Arroyo Valfondo & $1505 \pm 55$ & $1403 \pm 60$ \\
UZ5523/ETH34407 & UM 2 & AV-2 & Carbón & Arroyo Valfondo & $2745 \pm 55$ & $2848 \pm 55$ \\
UZ5519/ETH34403 & UM 2 & AG-12 & Carbón & Arroyo Grande & $3005 \pm 55$ & $3190 \pm 88$ \\
UZ5626/ETH35392 & UM 2 & AG-8 & Carbón & Arroyo Grande & $3270 \pm 55$ & $3502 \pm 68$ \\
UZ5216/ETH30527 & UM 2 & AG-6 & Carbón & Arroyo Grande & $4245 \pm 55$ & $4763 \pm 87$ \\
\hline
\end{tabular}

Debido al bajo número de ejemplares obtenidos en las muestras estudiadas, aquellas especies que aparecen representadas por más de 4 individuos por muestra se consideran "abundantes" en este trabajo.

Para efectuar la identificación taxonómica, a nivel de especie, de los ejemplares extraídos, se han seguido las clasificaciones definidas por Hartman \& Puri (1974) y Horne et al. (2002), completadas con los trabajos de Kempf (1980-1997), Meisch (2000) y Fuhrmann (2012). Para las especies del género Ilyocypris se han tenido en consideración las descripciones de Janz (1994), Karanovic \& Lee (2013) y Mazzini et al. (2014), mientras que para el género Paralimnocythere se ha seguido el trabajo de Martens (1992).

En los sedimentos fluvio-aluviales, como los que se estudian en este trabajo, es común el depósito de abundantes fragmentos de valvas de ostrácodos, fruto del transporte post-morten de las mismas por los flujos tractivos canalizados (e.g. Poquet \& Mesquita-Joanes, 2011). Estos fragmentos pueden ser transportados largas distancias, produciendo acumulaciones mezcladas con las asociaciones in-situ una vez que estos flujos se desbordan en la llanura de inundación y depositan el sedimento acarreado. De esta manera, la asociación estudiada no permitiría efectuar una reconstrucción paleoecológica fiable del ambiente de depósito. Esta situación se ha observado en el presente trabajo, puesto que en las 41 muestras estudiadas (Fig. 2) son abundantes los fragmentos de valvas de ostrácodos, inidentificables siquiera a nivel genérico.

Para evitar esta eventualidad, en este estudio únicamente se han considerado los ejemplares que no presentan evidentes signos de transporte (e.g. fracturas, roturas, abrasiones o superficies de erosión) para poder así realizar una interpretación adecuada de las condiciones ambientales en el momento de depósito. Además, la presencia de valvas y caparazones de ejemplares adultos y juveniles en la misma asociación, junto con una proporción similar de mudas en cada muestra, apoya la suposición de la ausencia de transporte en estos individuos, validando la reconstrucción paleoecológica realizada en este trabajo.

\section{Resultados}

En las 41 muestras estudiadas se han obtenido un total de 359 ostrácodos, identificándose 17 especies pertenecientes a 12 géneros (Tabla 2). La asignación de la especie Ilyocypris bradyi Sars se basa en la morfología externa de las valvas, ya que no ha sido posible la observación de criterios internos de las mismas. Externamente (ver Fig. 3), esta morfología es muy parecida a los ejemplares figurados por Fuhrmann (2012) y por Mazzini et al. (2014) como Ilyocypris bradyi Sars en diversos yacimientos centroeuropeos. En el caso del género Paralimnocythere, los ejemplares encontrados en las muestras estudiadas pertenecen a una especie, probablemente nueva, que aparece abundantemente en el Mioceno de la Cuenca del Ebro (e.g. Martínez-García et al., 2014) y es claramente distinta de Paralimnocythere psammophila (Flössner), por lo que se han clasificado como Paralimnocythere aff. psammophila (Tabla 2). Así, las especies más comunes en las muestras estudiadas son I. bradyi y Paralimnocythere aff. psammophila, estando acompañadas por Pseudocandona albicans (Brady) (Fig. 3; Tabla 2).

A continuación, se presenta de manera detallada la distribución de los ostrácodos obtenidos en cada una de las secciones estudiadas. 
Tabla 2.-Distribución estratigráfica, en valores absolutos, de las especies de ostrácodos identificadas en las muestras estudiadas de las secciones de Arroyo Grande (A) y Arroyo Valfondo (B). A la derecha se delimitan las Unidades Morfosedimentarias (UM) definidas

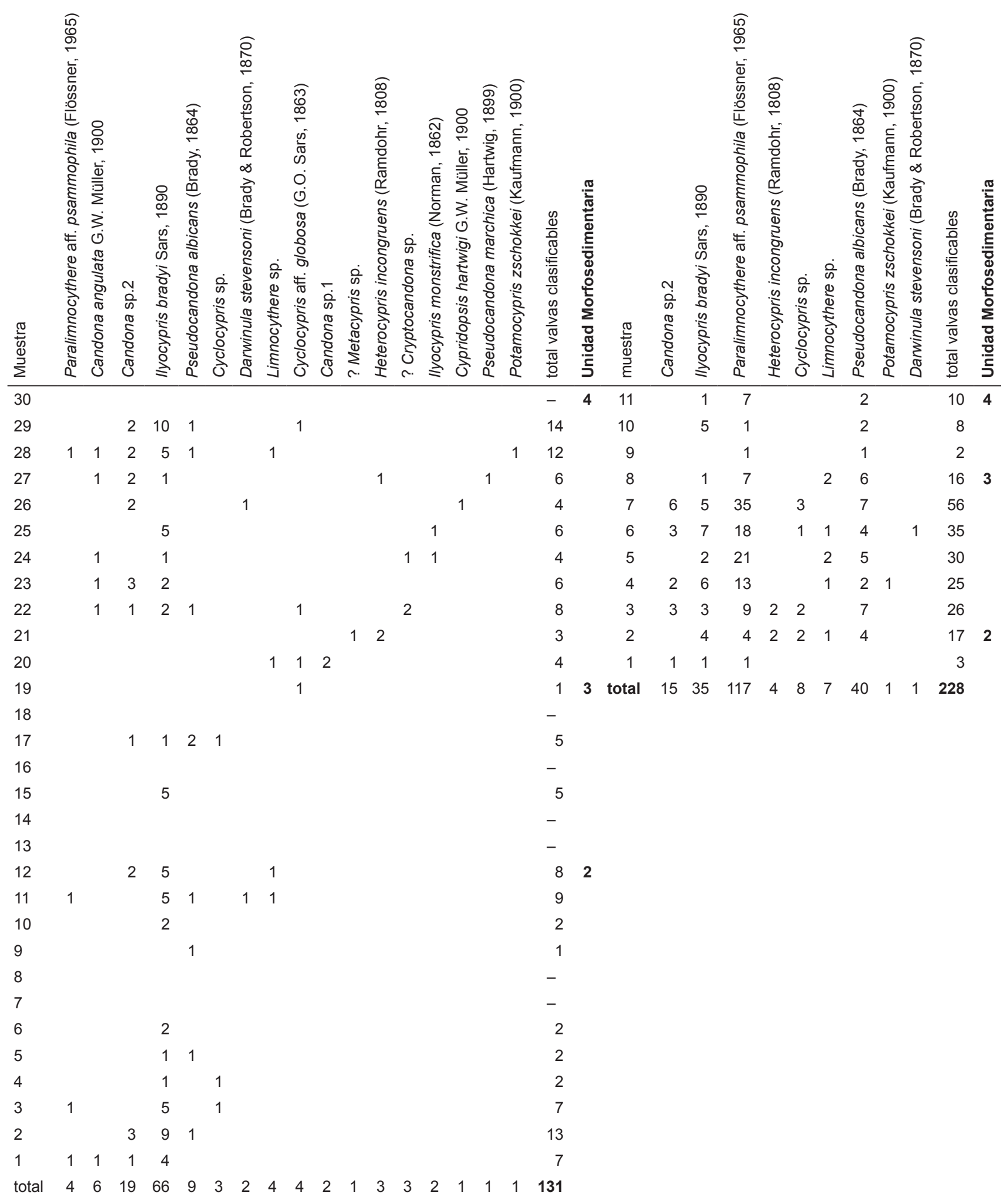




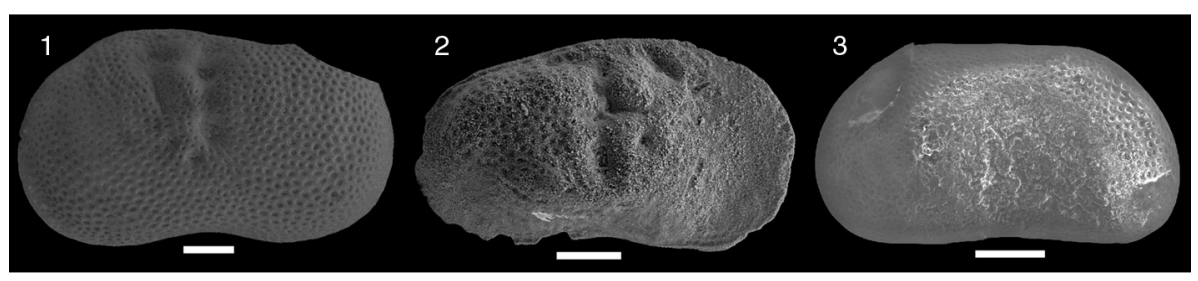

Fig. 3.-Fotografías MEB (Microscopio Electrónico de Barrido) de las especies más abundantes de ostrácodos identificadas en las muestras estudiadas. Todas las fotografías son vista externa; VD=valva derecha, $\mathrm{VI}=$ valva izquierda, $\mathrm{AG}-=\mathrm{Arroyo} \mathrm{Grande}, \mathrm{AV}-=\mathrm{Arroyo}$ Valfondo. Barra de escala=100 $\mu \mathrm{m}$. 1-llyocypris bradyi Sars, 1890, VI, AG-2; 2-Paralimnocythere aff. psammophila (Flössner, 1965), VD, AV-7; 3-Pseudocandona albicans (Brady, 1864), VD, AV-2.

\section{Sección de Arroyo Grande}

De las 30 muestras estudiadas, estratigráficamente sucesivas (Fig. 2A), siete de las mismas no presentaban ostrácodos clasificables (niveles $\mathrm{AG}-7,8,13$, $14,16,18$ y 30$)$. En el resto de los niveles analizados se han extraído un total de 131 ejemplares, pertenecientes a 17 especies (Tabla 2). Tanto la abundancia de ostrácodos como las especies más comunes identificadas en las tres Unidades Morfosedimentarias
(UM 2, UM 3, UM 4) descritas en esta sección, son diferentes (Fig. 4).

Unidad Morfosedimentaria UM $2(4763 \pm 87$ a $2848 \pm 55$ años cal. BP). Se identifica en la base de la sección e incluye desde el nivel AG-1 al nivel AG-12, donde la especie más abundante es I. bradyi, estando acompañada en algunos niveles por P. albicans (Fig. 4; Tabla 2).

Unidad Morfosedimentaria UM $3(2848 \pm 55$ a $836 \pm 65$ años cal. BP). Se reconoce en la parte central

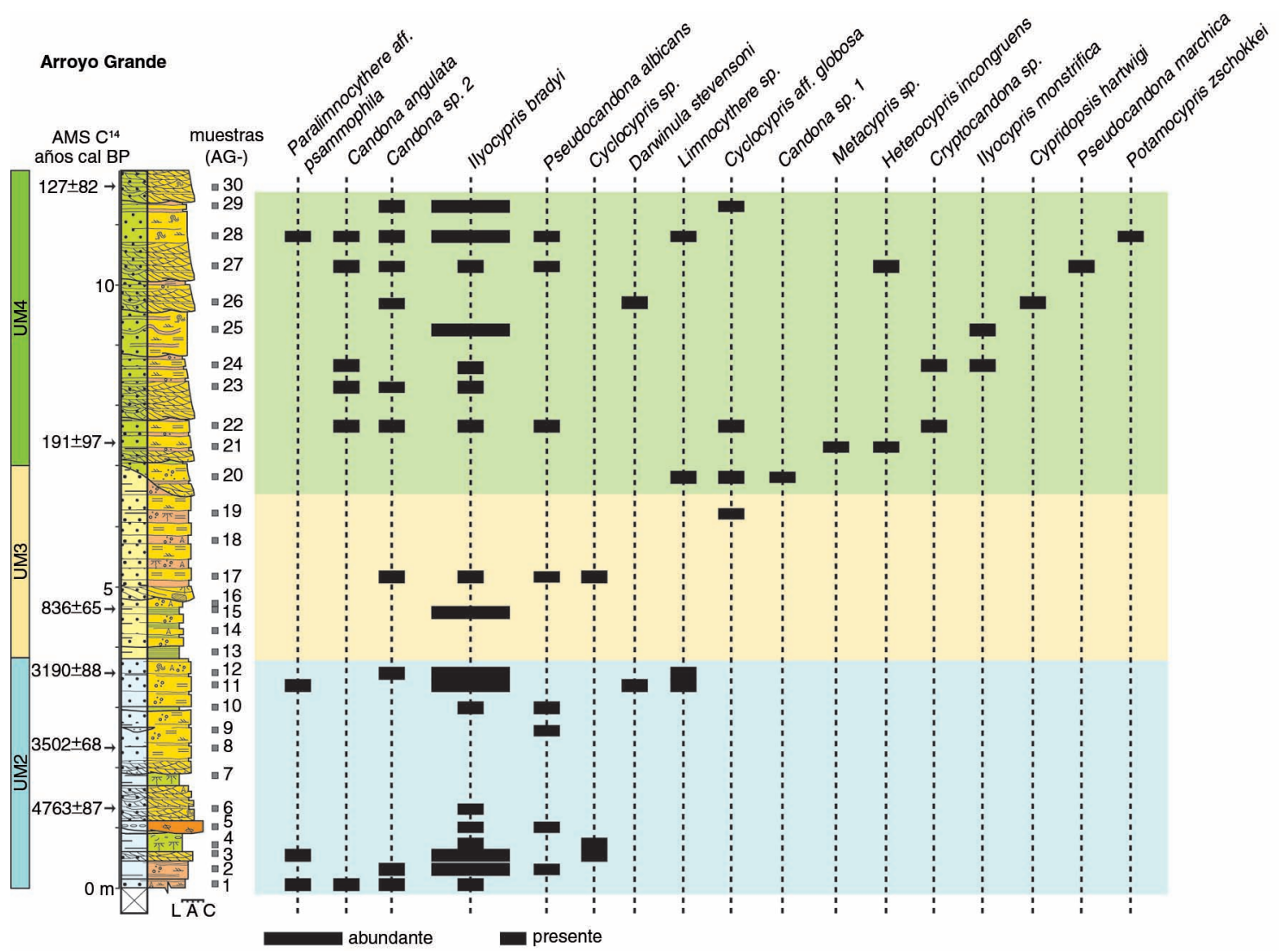

Fig. 4.-Distribución estratigráfica de las especies de ostrácodos identificadas en las muestras estudiadas de la sección de Arroyo Grande, separadas en abundantes y presentes. Para leyenda de la columna, ver Fig. 2. 
de la sección y engloba las muestras desde AG-13 a AG-19 (Fig. 4). Esta unidad es muy pobre en ostrácodos, encontrándose ejemplares clasificables únicamente en tres de las muestras estudiadas (AG-15, 17 y 19), donde se registra una mezcla de escasos individuos pertenecientes a I. bradyi o P. albicans junto con otros del género Cyclocypris (Fig. 4; Tabla 2).

Unidad Morfosedimentaria UM 4 (191 \pm 97 a $127 \pm 82$ años cal. BP). Se define hacia el techo de la sección (muestras desde AG-20 hasta AG-30) (Fig. 4). En la base de este intervalo aparecen algunos ejemplares de Candona sp. 1 (nivel AG-20) y Heterocypris incongruens (Ramdohr) (nivel AG-21), mientras que en el resto de las muestras estudiadas que componen esta UM 4 es más abundante I. bradyi, estando acompañada por Candona sp. 2 (Fig. 4; Tabla 2).

\section{Sección de Arroyo Valfondo}

Se han estudiado 11 muestras recogidas a lo largo de esta sección (Fig. 2B), obteniéndose 228 ejemplares de ostrácodos agrupados en 9 especies (Tabla 2).
De nuevo se observan diferencias en la abundancia relativa de las especies más comunes en las tres Unidades Morfosedimentarias identificadas (UM 2, UM 3, UM 4) (Fig. 5).

Unidad Morfosedimentaria UM $2(4763 \pm 87$ a $2848 \pm 55$ años cal. BP). Se localiza en la base de la columna, englobando únicamente dos de los niveles muestreados (AV-1 y 2), donde aparece como especie más abundante $I$. bradyi, junto con Paralimnocythere aff. psammophila y P. albicans (Fig. 5; Tabla 2).

Unidad Morfosedimentaria UM $3(2848 \pm 55$ a $836 \pm 65$ años cal. BP). Es la que presenta un mayor espesor en la sección de Arroyo Valfondo (Fig. 5) e incluye los niveles de AV-3 a AV-8. La especie más abundante en todo este intervalo es Paralimnocythere aff. psammophila, aunque también se encuentran ejemplares de P. albicans e I. bradyi a lo largo de esta unidad (Fig. 5; Tabla 2).

Unidad Morfosedimentaria UM4(191 \pm 97 a $127 \pm 82$ años cal. BP). Se identifica a techo de la sección y engloba los niveles AV-9 a AV-11 (Fig. 5). En este último intervalo disminuye el número de ostrácodos,

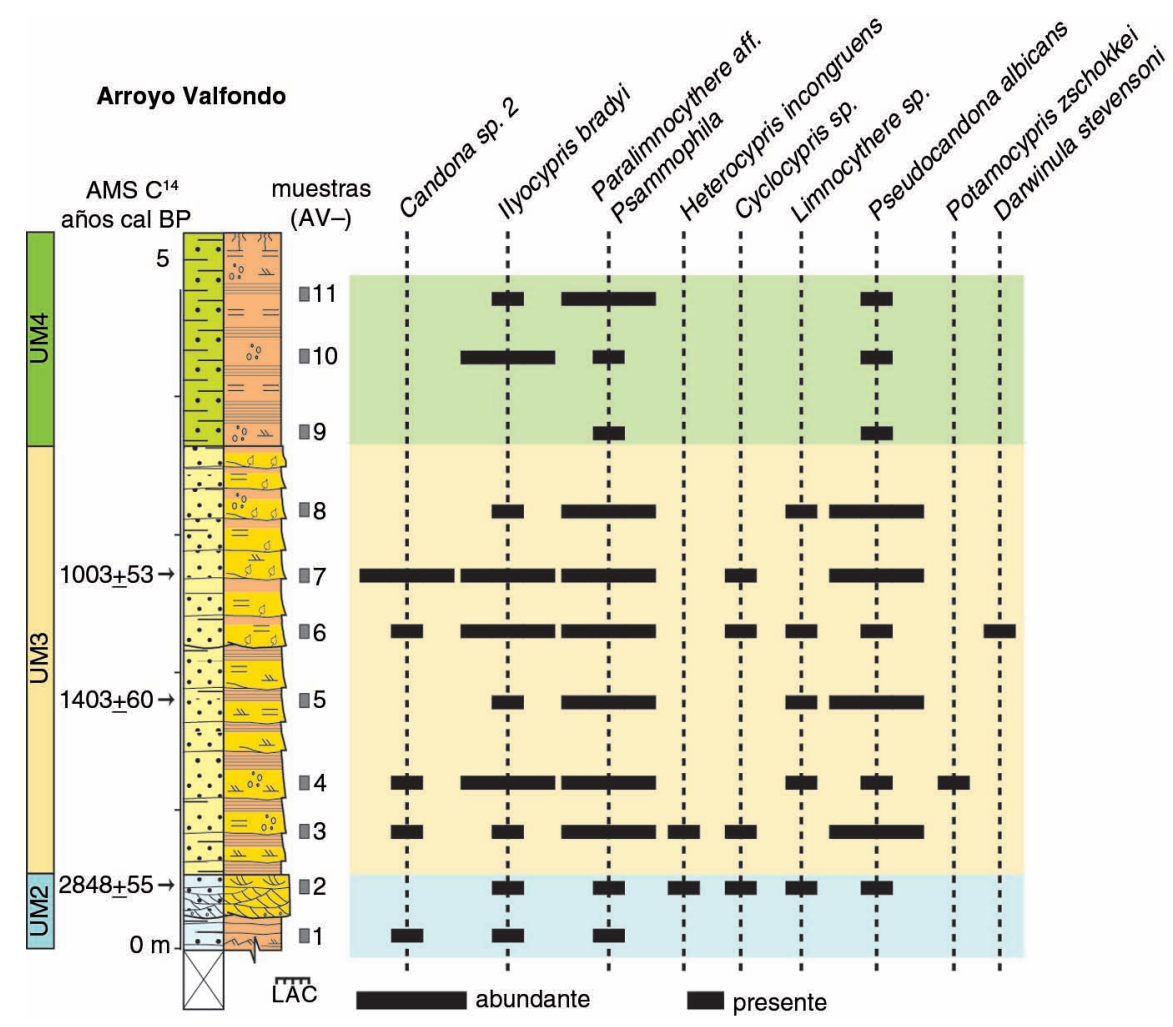

Fig. 5.-Distribución estratigráfica de las especies de ostrácodos identificadas en las muestras estudiadas de la sección de Arroyo Valfondo, separadas en abundantes y presentes. Para leyenda de la columna, ver Fig. 2. 
estando presentes las especies $I$. bradyi y P. albicans junto con Paralimnocythere aff. psammophila, todas ellas con escasos ejemplares.

\section{Discusión}

Aludiendo a la asociación de ostrácodos identificada en las muestras estudiadas, destaca la presencia de manera más o menos continuada a lo largo de todo el registro de I. bradyi, Paralimnocythere aff. psammophila y, en menor medida, $P$. albicans. Sin embargo, se observan variaciones en la abundancia relativa de dichas especies en cada una de las Unidades Morfosedimentarias estudiadas, que parecen indicar cambios internos en el régimen hidrodinámico del sistema aluvial a lo largo del Holoceno final, posiblemente relacionados con alteraciones climáticas. De esta manera, y de acuerdo a los requisitos ecológicos de dichas especies de ostrácodos (Tabla 3), se propone una reconstrucción paleoambiental regional de la zona de estudio durante el Holoceno final, complementando reconstrucciones previas como las de Sancho et al. (2008), Murelaga et al. (2012) y Bastida et al. (2013).

\section{$4763 \pm 87$ a $2848 \pm 55$ años cal. BP}

Los depósitos sedimentarios correspondientes a este intervalo temporal (UM 2) presentan un registro más amplio en la sección de Arroyo Grande (Fig. 2A). La asociación de ostrácodos identificada en las muestras recogidas en este intervalo está dominada por I. bradyi (Fig. 4; Tabla 2). Esta especie es indicativa de ambientes con agua corriente, tales como pequeños arroyos, riachuelos o manantiales helocrenos y reocrenos (Henderson, 1990; Roca \& Baltanás, 1993; Külköylüoğlu, 1999; Wu et al., 2001; Mezquita et al., 2005), aunque también puede aparecer en charcas temporales o ambientes lacustres de aguas estancadas (Henderson, 1990) asociada a la entrada ocasional de agua corriente desde arroyos cercanos (Curry, 1999). A lo largo de la UM 2, y en especial a techo de dicha unidad en Arroyo Valfondo (Figs. 4 y 5; Tabla 2), aparece $P$. albicans. Esta especie es típica de bordes de lagos con cierta corriente interna del agua (Meisch, 2000; Mezquita et al., 2005) (Tabla 3), por lo que su aparición confirmaría la presencia de aguas corrientes en esta área para este período.
Esta UM 2 está constituida por varias secuencias solapadas de canales arenosos y depósitos de desbordamiento (ver Fig. 2A) indicativos del desarrollo de un sistema fluvial de canales activos y áreas encharcadas (Murelaga et al., 2012). Estas secuencias sedimentarias identificadas en este intervalo temporal en Bardenas Reales de Navarra, podrían representar la respuesta de pequeños sistemas fluviales ante unas condiciones ambientales caracterizadas por una distribución relativamente regular de las precipitaciones, unas laderas fitoestabilizadas y escasez de eventos hidrológicos extremos, lo que se traduce en una cierta estabilidad geomorfológica y en el desarrollo de paleosuelos (Murelaga et al., 2012). Los estudios palinológicos efectuados en Bardenas Reales de Navarra, definen el desarrollo de un bosque ribereño relacionado con flujos de agua permanente en la depresión de Bardena Blanca en 3495 35 años BP (Iriarte \& Meaza, 1996), en torno a la Época Fría del Hierro. Así mismo, los estudios faunísticos (gasterópodos) e isotópicos previamente realizados, permiten inferir una tendencia hacia condiciones ambientales húmedas y frías durante este periodo en esta zona (Murelaga et al., 2012). Por otro lado, Thorndycraft \& Benito (2006) han descrito un descenso en las avenidas durante el final del Holoceno medio en la Península Ibérica que apunta a una fase de cierta estabilidad hidrológica.

La interpretación paleoambiental establecida a partir de la asociación de ostrácodos identificada en este trabajo es concordante con estas observaciones previas, puesto que define el desarrollo de un sistema de pequeños arroyos o riachuelos con agua corriente en una llanura de inundación entre $4763 \pm 87$ y $2848 \pm 55$ años cal. BP (UM 2).

\section{$2848 \pm 55$ a $836 \pm 65$ años cal. BP}

A pesar de que ambas secciones estudiadas presentan una potencia similar de sedimentos depositados durante este intervalo temporal (UM 3), es en las muestras recogidas en Arroyo Valfondo (Fig. 2B) donde se ha encontrado un mayor número de ostrácodos clasificables.

En todos los niveles correspondientes a este intervalo, Paralimnocythere aff. psammophila es la especie dominante (Fig. 5; Tabla 2). Considerando unos requisitos ecológicos para esta especie similares a los descritos para P. psammophila, es posible efectuar 
Tabla 3.-Resumen de los principales requisitos ecológicos de las especies de ostrácodos identificadas. Referencias: ${ }^{(1)}$ Meisch (2000); ${ }^{(2)}$ Horne et al. (2012); ${ }^{(3)}$ Baltanás et al. (1996); ${ }^{(4)}$ Mezquita et al. (2005); ${ }^{(5)}$ Margalef (1953); ${ }^{(6)}$ McGregor (1969); ${ }^{(7)}$ Ranta (1979); ${ }^{(8)}$ Niinemets (1999); ${ }^{(1)}$ Griffiths \& Butlin (1994); ${ }^{(10)}$ Henderson (1990); ${ }^{(11)}$ Curry (1999); ${ }^{(12)}$ Mezquita et al. (1999); ${ }^{(13)}$ Gascón et al. (2009); ${ }^{(14)}$ Mezquita et al. (1996); ${ }^{(15)}$ Diebel \& Pietrzeniuk (1990)

\begin{tabular}{|c|c|c|c|c|c|c|}
\hline Especies & profundidad & hidrodinamismo & temperatura & salinidad & contenido en $\mathrm{Ca}^{2+}$ & comentarios \\
\hline $\begin{array}{l}\text { Candona } \\
\text { (género) }\end{array}$ & - & - & $\begin{array}{l}\text { estenotérmica de } \\
\text { aguas frías }{ }^{(1)}\end{array}$ & - & $\begin{array}{l}\text { tolera aguas } \\
\text { altamente } \\
\text { alcalinas }^{(1)}\end{array}$ & - \\
\hline $\begin{array}{l}\text { Candona } \\
\text { angulata }\end{array}$ & $\begin{array}{l}\text { entre } 5-220 \mathrm{~m} \\
\text { de profundidad } \\
\text { (principalmente }\end{array}$ & $\begin{array}{l}\text { aguas fluyentes } \\
\text { y estancadas } \\
{\text { (reoeuriplástica) })^{(1)}}^{\text {(reous }}\end{array}$ & $\begin{array}{l}\text { mesotermófila } \\
\text { (aguas frías y }_{\text {cálidas) }^{(1,2)}}\end{array}$ & $\begin{array}{l}\text { oligohalina a } \\
\text { mesohalina } \\
(0,2-14 \% \circ)^{(1)}\end{array}$ & $\begin{array}{l}\text { mesotitanófila } \\
\text { a polititanófila } \\
(18->72 \mathrm{mg} /)^{(1)}\end{array}$ & $\begin{array}{l}\text { También aparece en aguas de baja salinidad de } \\
\text { lagos, lagunas costeras, acequias y desembocadura } \\
\text { de ríos }{ }^{(1)} \text {. }\end{array}$ \\
\hline
\end{tabular}

Cyclocypris

(género)

Cyclocypris

globosa

Cypridopsis

hartwigi

Darwinula

stevensoni

zonas

lacustres poco

aguas

profundas

borde somero

de lagos

(entre 0-85 m

principalmente

$<9 \mathrm{~m}^{(1)}$

Heterocypris

incongruens

\section{aguas corrientes
(mesoreófila) $^{(1,4)}$}

mesotermófila

(aguas frías y cálidas $)^{(1,2)}$, óptimo $14,8^{\circ} \mathrm{C}^{(4)}$ aguas templadas a cálidas $^{(2,4)}$, óptimo

$15^{\circ} \mathrm{C}^{(4)}$

termoeuriplástica ${ }^{(1,2)}$ (euritérmica $^{(6,7)}$ ), de turbulencia y oca corriente

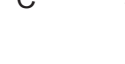

altamente sensitivo
ante cambios en la salinidad del medio ${ }^{(1)}$

$$
\text { - } \text { bajo }^{(1)}
$$

aguas ligeramente saladas $^{(5)}$, óptimo $3,19 \mu \mathrm{s} / \mathrm{cm}^{(4)}$ $3,19 \mu \mathrm{s} / \mathrm{cm}$ $\left(5-18 \% o^{(1)}\right)$, óptimo $3,09 \mu \mathrm{s} / \mathrm{cm}^{(4)}$

timo
alcalinidad $^{(4)}$

probablemente

titanoeuriplástica

$\left(8->72 \mathrm{mg} / \mathrm{l}^{(1)}\right.$ aguas con baja

En la Península Ibérica aparece restringido a

ambientes lacustres (aguas estancadas) de zonas frías (N Península Ibérica o lagunas de montaña) $)^{(3,4)}$.

Típica de turberas, su presencia y abundancia se asocian con bajo $\mathrm{pH}$, alto contenido en materia orgánica en el sedimento y bajo oxígeno disuelto ${ }^{(1)}$.

Aguas expuestas a la luz solar y ricas en vegetación, temporales o permanentes ${ }^{(1)}$, con $\mathrm{pH}$ básico (óptimo 8,01 ) y bien oxigenadas (óptimo $8 \mathrm{mg} / \mathrm{l}^{(4)}$.

Común en aguas permanentes de lagos, charcas y arroyos con sustratos arenosos o fangosos ${ }^{(1)}$. También aparece en aguas intersticiales ${ }^{(1)}$. Prefiere el sustrato rico en materia orgánica ${ }^{(1)}$. El oxígeno es un factor limitante (reproducción en aguas frías bien oxigenadas) $)^{(8)}$, óptimo $8,4 \mathrm{mg} / \mathrm{I}^{(4)}$. Abundante en zonas litorales de lagos oligotróficos ${ }^{(9)}$.

oligohalina $(0,5-5 \%$ ), titanoeuriplástica Prefiere las charcas de aguas temporales y tolera grandes tolera grandes salinidad $^{(1)}$, óptimo $3,06 \mu \mathrm{s} / \mathrm{cm}^{(4)}$ pequeños cuerpos de aguas estables ${ }^{(1,4)}$ y sustrato fangoso sin macrófitas ${ }^{(1)}$. Se ha encontrado en aguas estancadas y aguas subterráneas, siendo una especie rara en cuerpos de agua grandes y estables $^{(1)}$. Aunque prefiere aguas bien oxigenadas (óptimo $8 \mathrm{mg} / \mathrm{l}^{(4)}$, puede colonizar aguas

fuertemente eutrofizadas, con bajo contenido en oxígeno y alto contenido en polución orgánica ${ }^{(1)}$. 
Tabla 3.- (continued)

\begin{tabular}{|c|c|c|c|c|c|c|}
\hline Especies & profundidad & hidrodinamismo & temperatura & salinidad & contenido en $\mathrm{Ca}^{2+}$ & comentarios \\
\hline Ilyocypris bradyi & $\begin{array}{l}\text { entre } 0-4 m \text { de } \\
\text { profundidad }^{(8)}\end{array}$ & $\begin{array}{l}\text { aguas corrientes } \\
(5,10) \text {, también } \\
\text { aparece } \\
\text { en aguas } \\
\text { estancadas } \\
\text { con entrada } \\
\text { ocasional } \\
\text { de agua } \\
\text { corriente }^{(10,11)}\end{array}$ & $\begin{array}{l}\text { aguas frías a } \\
\text { cálidas }^{(5,8)} \text {, óptimo } \\
14,5^{\circ} \mathrm{C}^{(4)}\end{array}$ & $\begin{array}{l}\text { oligohalina } \\
(0,3-4,4 \% \circ)^{(8)} \text {, óptimo } \\
3,01 \mu \mathrm{s} / \mathrm{cm}^{(4)}\end{array}$ & $\begin{array}{l}\text { mesotitanófila } \\
(18->72 \mathrm{mg} /)^{(8)}\end{array}$ & $\begin{array}{l}\text { Aunque prefiere aguas básicas (pH óptimo } 7,8) \text { y } \\
\text { bien oxigenadas (óptimo } 8,8 \mathrm{mg} / \mathrm{l})^{(4)} \text {, es capaz de } \\
\text { tolerar concentraciones bajas de oxígeno }(3 \mathrm{mg} /)^{(12)} \text {. }\end{array}$ \\
\hline $\begin{array}{l}\text { Ilyocypris } \\
\text { monstrifica }\end{array}$ & - & - & euritérmica $^{(1,2)}$ & baja salinidad $^{(1)}$ & - & Vive en medios lacustres $^{(1)}$ \\
\hline $\begin{array}{l}\text { Limnocythere } \\
\text { (género) }\end{array}$ & $\begin{array}{l}\text { borde de lagos } \\
\text { someros }^{(1)}\end{array}$ & - & - & $\begin{array}{l}\text { aguas ligeramente } \\
\text { saladas }^{(1)}\end{array}$ & $\begin{array}{l}\text { aguas altamente } \\
\text { alcalinas }^{(1)}\end{array}$ & - \\
\hline $\begin{array}{l}\text { Paralimnocythere } \\
\text { psammophila }\end{array}$ & $\begin{array}{l}\text { borde somero } \\
\text { de } \operatorname{lagos}^{(1)}\end{array}$ & $\begin{array}{l}\text { aguas } \\
\text { estancadas }^{(4)}\end{array}$ & $\begin{array}{l}\text { aguas frías a } \\
\text { cálidas }^{(2,4)} \text {, óptimo } \\
13,8{ }^{\circ} \mathrm{C}^{(4)}\end{array}$ & $\begin{array}{l}\text { aguas ligeramente } \\
\text { saladas, óptimo } 2,86 \\
\mu \mathrm{s} / \mathrm{cm}^{(4)}\end{array}$ & aguas alcalinas $^{(4)}$ & 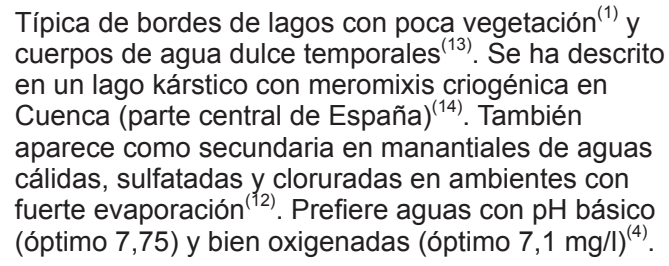 \\
\hline $\begin{array}{l}\text { Potamocypris } \\
\text { zschokkei }\end{array}$ & - & 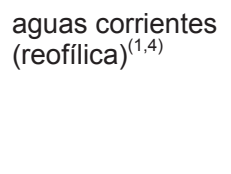 & $\begin{array}{l}\text { aguas frías a } \\
\text { cálidas }^{(2)} \text {, prefiere } \\
\text { aguas frías }{ }^{(1)} \\
\text { óptimo } 10,2^{\circ} \mathrm{C}^{(4)}\end{array}$ & $\begin{array}{l}\text { aguas ligeramente } \\
\text { saladas, óptimo } \\
2,66 \mu \mathrm{s} / \mathrm{cm}^{(4)}\end{array}$ & $\begin{array}{l}\text { aguas altamente } \\
\text { alcalinas }^{(4)}\end{array}$ & $\begin{array}{l}\text { Prefiere arroyos con fondo fangoso }{ }^{(1)} \text {. Aparece en } \\
\text { aguas intersticiales y, posiblemente, en cuevas o } \\
\text { pasajes subterráneos (estigofílica) }{ }^{(1)} \text {. Prefiere aguas } \\
\text { básicas }(\mathrm{pH} \text { óptimo } 7,53) \text { y bien oxigenadas (óptimo } \\
9,4 \mathrm{mg} / \mathrm{l})^{(4)} \text {. }\end{array}$ \\
\hline $\begin{array}{l}\text { Pseudocandona } \\
\text { albicans }\end{array}$ & $\begin{array}{l}\text { borde somero } \\
\text { de lagos }^{(1)}\end{array}$ & $\begin{array}{l}\text { aguas fluyentes } \\
\text { y estancadas } \\
\text { (reoeuriplástica) } \\
(1) \text {, es común en } \\
\text { aguas corrientes } \\
\text { (mesoreófila) }^{(1,4)}\end{array}$ & $\begin{array}{l}\text { mesotermófila } \\
\text { (aguas frías y } \\
\text { cálidas) }{ }^{(1,2,15)} \\
\text { óptimo } 14,3^{\circ} \mathrm{C}^{(4)}\end{array}$ & $\begin{array}{l}\text { oligohalina a } \\
\text { mesohalina }(<5,5 \%) \\
\text { (1), óptimo } 3,28 \mu \mathrm{s} / \\
\mathrm{cm}^{(4)}\end{array}$ & $\begin{array}{l}\text { titanoeuriplástica } \\
(8->72 \mathrm{mg} /)^{(1)}\end{array}$ & $\begin{array}{l}\text { Prefiere sustrato limo-arcilloso con abundante } \\
\text { vegetación }{ }^{(15)} \text {. Se ha encontrado en manantiales } \\
\text { sulfurosos }^{(1)} \text {. Prefiere aguas con pH básico (óptimo } \\
7,95) \text { y bien oxigenadas (óptimo } 8,1 \mathrm{mg} / \mathrm{l}^{(4)} \text {. }\end{array}$ \\
\hline $\begin{array}{l}\text { Pseudocandona } \\
\text { marchica }\end{array}$ & $\begin{array}{l}\text { borde somero } \\
\text { de lagos }{ }^{(1)}\end{array}$ & $\begin{array}{l}\text { aguas con algo } \\
\text { de turbulencia y } \\
\text { poca corriente } \\
\text { (oligoreófila) }^{(1)}\end{array}$ & $\begin{array}{l}\text { aguas frías a } \\
\text { cálidas }^{(2)}, \text { prefiere } \\
\text { aguas cálidas } \\
\text { (politermófila) }^{(1)}\end{array}$ & oligohalina $(<4 \% 0)^{(1)}$ & $\begin{array}{l}\text { titanoeuriplástica } \\
\left(8->72 \mathrm{mg} / \mathrm{l}^{(1)}\right.\end{array}$ & $\begin{array}{l}\text { Típica de ambientes acuáticos continentales muy } \\
\text { variados: pequeños cuerpos de aguas permanentes } \\
\text { y/o temporales, hábitats intersticiales de arroyos, } \\
\text { ríos, manantiales y campos de cultivo }{ }^{(1)} \text {. Presente } \\
\text { en aguas con abundante vegetación acuática }{ }^{(9)} \text {. } \\
\text { Probablemente euriplástica para el } \text { pH }^{(1)} \text {. }\end{array}$ \\
\hline
\end{tabular}


una interpretación paleoambiental para este intervalo temporal. Esta especie es típica de cuerpos de agua dulce temporales o zonas litorales de ambientes lacustres sin apenas corrientes de agua (Meisch, 2000; Mezquita et al., 2005; Gascón et al., 2009) (Tabla 3). Por tanto, implicaría el establecimiento de un medio acuático efímero y de aguas estancadas. La aparición, tanto en la Sección de Arroyo Grande como en la de Arroyo Valfondo, de ejemplares de I. bradyi y de P. albicans, especies características de aguas corrientes, indicaría episodios esporádicos de precipitaciones irregulares, tal vez torrenciales, que aumentarían el hidrodinamismo en dichos cuerpos de agua estancada.

Los materiales que conforman la UM 3 se organizan en secuencias arenosas granodecrecientes, que presentan yeso, bioturbaciones y acumulaciones de gasterópodos y restos vegetales en la base de cada una de ellas (ver Fig. 2B). Esta unidad se interpreta como un periodo de elevada tasa de actividad aluvial, relacionada con lluvias esporádicas y torrenciales en condiciones climáticas más áridas que las acontecidas durante el intervalo temporal previo (Murelaga et al., 2012). En valles holocenos de la parte central de la Cuenca del Ebro se han descrito secuencias aluviales similares (Peña et al., 2004; Constante et al., 2010). Además, en materiales lacustres muestreados en las Sierras Exteriores de los Pirineos, Morellón et al. (2009) identifican una fase árida acontecida entre 870 y 750 años cal. BP.

El intervalo temporal de este depósito aluvial coincide con un incremento en la erosión de laderas ocurrida durante los periodos Romano y post-Romano, dentro de una tendencia general hacia la aridificación que culmina en el "Periodo Cálido Medieval" o "Anomalía Climática Medieval" (Moreno et al., 2012). Durante este periodo (datado aproximadamente en 800-1200 d.C.) las temperaturas de algunas zonas europeas alcanzaron los valores máximos de los últimos 4000 años (Barclay et al., 2009). Estas condiciones climáticas explicarían la disminución en el hidrodinamismo del sistema aluvial en este periodo con respecto al intervalo previo en esta área, tal y como han evidenciado las especies de ostrácodos identificadas. Por otro lado, en la parte central de la Cuenca del Ebro se ha constatado que estos procesos aluviales pueden acentuarse localmente debido a la actividad humana, ya sea por un aumento del pastoreo o por la deforestación (Iriarte \& Meaza, 1996; Peña et al., 2004; González-Sampériz et al., 2009), por lo que no se puede despreciar la influencia antrópica como explicación de la variación en las condiciones del medio aluvial detectada en este estudio.

\section{$191 \pm 97$ a $127 \pm 82$ años cal. BP}

Este intervalo temporal (UM 4) presenta una mayor potencia de sedimentos depositados en la sección de Arroyo Grande (Fig. 2A). De manera general, la asociación específica de ostrácodos identificada en esta unidad está dominada por I. bradyi (Fig. 4; Tabla 2), lo que implicaría, nuevamente, el establecimiento de un medio aluvial con agua corriente en el Reciente. La presencia de algunos ejemplares de otras especies menos abundantes a lo largo de esta Unidad Morfosedimentaria definiría cambios de menor escala temporal dentro de este intervalo. Así, en la base de la sección de Arroyo Grande aparece H. incongruens (Tabla 2), lo que implica el desarrollo de pequeños cuerpos de agua temporales con cierta corriente interna (Meisch, 2000; Mezquita et al., 2005). Por su parte, a techo de Arroyo Valfondo abunda Paralimnocythere aff. psammophila (Tabla 2), indicando la presencia de cuerpos de agua estancada en la llanura de inundación aluvial.

A partir del análisis de las asociaciones de gasterópodos y la señal isotópica de sus conchas, Murelaga et al. (2012) identificaron el establecimiento de unas condiciones relativamente frías y con alternancias de fases húmedas y secas en el periodo Reciente en Bardenas Reales de Navarra. Estos autores aluden a la variabilidad climática acontecida durante la "Pequeña Edad de Hielo" (Little Ice Age, LIA) como posible causante del incremento en las condiciones de frío y los cambios hidrológicos extremos de menor escala deducidos durante este intervalo temporal en esta área. Este evento, datado aproximadamente en 1500-1850 d.C., consiste en un primer avance de los glaciares continentales entre 1180-1320 d.C. (Barclay et al., 2009), seguido por dos etapas frías principales, de aproximadamente un siglo de duración cada una de ellas, acontecidas en los siglos XVII y XIX de nuestra era, con dos fases relativamente cálidas ocurridas en los siglos XVI y XVIII (Bradley \& Jones, 1992; Barclay et al., 2009). Las fases frías supusieron 
un nuevo avance de los glaciares en Europa, Asia y Norteamérica, con una expansión del hielo oceánico en el Atlántico Norte (Lamb, 1982). Sin embargo, algunos autores han puesto en duda que se trate de un evento global (e.g. Bradley \& Jones, 1992). En el norte de la Península Ibérica, la LIA se caracteriza por un incremento en la humedad y un descenso en la temperatura (Valero-Garcés et al., 2008; Morellón et al., 2009), con una alta variabilidad climática de menor escala que incluye fases de inundaciones extremas y periodos de sequía (e.g. Álvarez Vázquez, 1986; Benito et al., 1996; Saz, 2003). Estas condiciones climáticas serían las causantes de la influencia de un agua corriente, posiblemente asociada a arroyos o riachuelos de pequeña entidad, alternante con momentos en los que se desarrollan cuerpos de agua efímeros y estancados durante el periodo Reciente en Bardenas Reales de Navarra, tal y como se ha constatado en la reconstrucción paleoambiental efectuada a partir de las asociaciones de ostrácodos. Sin embargo, tal y como sucedía en el intervalo temporal previo, no se puede descartar la posible influencia antrópica, consistente principalmente en prácticas de deforestación, durante la LIA en esta área (Murelaga et al., 2012).

\section{Conclusiones}

En este trabajo se ha efectuado una reconstrucción paleoambiental de las secuencias aluviales de Bardenas Reales de Navarra durante el Holoceno final, a partir de las asociaciones de ostrácodos identificadas en las muestras recogidas en las secciones estratigráficas de Arroyo Grande y Arroyo Valfondo controladas cronológicamente mediante radiocarbono.

Para ello se han estudiado 41 muestras, de las que se han extraído 359 ejemplares de ostrácodos pertenecientes a 17 especies, siendo las más comunes a lo largo de todo el registro I. bradyi, Paralimnocythere aff. psammophila y P. albicans. De acuerdo a la variación en la abundancia relativa de dichas especies en la vertical de ambas secciones, se han podido registrar los siguientes cambios hidrodinámicos en este ambiente aluvial:

Debido al predominio de I. bradyi, se ha constatado el desarrollo de un sistema aluvial conformado por pequeños arroyos o riachuelos de agua corriente desde $4763 \pm 87$ hasta $2848 \pm 55$ años cal. BP, posiblemente generado en llanuras de inundación con colonización vegetal y desarrollo de paleosuelos. Entre $2848 \pm 55$ y $836 \pm 65$ años cal. BP, en la asociación de ostrácodos identificada la especie más abundante es Paralimnocythere aff. psammophila, lo que define el establecimiento de cuerpos efímeros de agua dulce y estancada, probablemente relacionados con un periodo de elevada tasa de actividad aluvial en condiciones climáticas más áridas correlacionables con la Anomalía Climática Medieval. Por último, en el

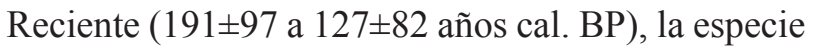
más común es $I$. bradyi, lo que permite inferir la instauración de un medio aluvial con mayor hidrodinamismo que en el intervalo anterior, correlacionable con la Pequeña Edad del Hielo.

Los ostrácodos se han revelado como útiles marcadores de los cambios ambientales de alta frecuencia acontecidos en esta zona semiárida durante el Holoceno final. Esto ha permitido complementar los estudios paleoambientales previos efectuados en esta área, así como realizar una comparativa a mayor escala con otros registros climáticos continentales holocenos de la Península Ibérica. En general, la evolución paleoambiental aquí deducida presenta una buena correlación con las variaciones climáticas rápidas acontecidas durante el Holoceno, que han sido previamente descritas y analizadas en el NE Peninsular.

\section{AGRADECIMIENTOS}

Los autores quieren agradecer la inestimable ayuda de Alejandro Urmeneta, de la Comunidad de Bardenas Reales de Navarra, en la recogida y procesado de las muestras estudiadas. También quieren agradecer a dos revisores anónimos sus sugerencias y comentarios, que han ayudado a mejorar sustancialmente este trabajo. Este trabajo se ha financiado con los proyectos GIU12/35 de la UPV/ EHU, HAR2014-53536-P y CGL2009-10455/BTE del Ministerio de Economía y Competitividad, una beca predoctoral (ref. BFI2012-118) del Gobierno Vasco (O. Suarez-Hernando), una beca postdoctoral (Contratación para la especialización de personal investigador doctor) de la UPV/EHU (B. Martínez-García) y por el Gobierno de Aragón y Fondo Social Europeo (Grupos PaleoQ y Análisis de cuencas sedimentarias continentales).

\section{Referencias}

Álvarez Vázquez, J.A. (1986). Drought and rainy periods in the Province of Zamora in the 17th, 18th and 19th centuries. In: Quaternary Climate in the Western 
Mediterranean (López Vera, F., Ed.). Universidad Autónoma de Madrid, Madrid, 221-233.

Baltanás, A.; Beroiz, B. \& López, A. (1996). Lista faunística y bibliográfica de los ostrácodos no-marinos (Crustacea, Ostracoda) de la Península Ibérica, Islas Baleares e Islas Canarias. Listas de la flora y fauna de las aguas continentales de la Península Ibérica, volumen 12. Asociación Española de Limnología, Madrid, $71 \mathrm{pp}$.

Barclay, D.J.; Wiles, G.C. \& Calkin, P.E. (2009). Holocene glacier fluctuations in Alaska. Quaternary Science Reviews, 28: 2034-2048. http://dx.doi.org/10.1016/j. quascirev.2009.01.016

Bastida, J.; Osácar, M.C.; Sancho, C. \& Muñoz, A. (2013). Environmental changes during the Upper PleistoceneHolocene in Mediterranean NE Spain as recorded by the mineralogy and geochemistry of alluvial records. Quaternary International, 302: 3-19. http://dx.doi.org/ 10.1016/j.quaint.2013.02.041

Benito, G.; Machado, M.J. \& Pérez-González, A. (1996). Climate change and flood sensitivity in Spain. In: Global Continental Changes: The Context of Palaeohydrology (Branson, J.; Brown, A.G. \& Gregory, K.J., Eds.). Geological Society of London Special Publication, 115, London, 85-98. http://dx.doi.org/10.1144/ GSL.SP.1996.115.01.08

Boomer, I.; Horne, D.J. \& Slipper, I.J. (2003). The use of ostracods in palaeoenvironmental studies, or what can you do with an ostracod shell?. In: Bridging the gap: trends in Ostracode biological and geological sciences (Park, L.E.; Smith, A.J., Eds.). Paleontological Society Papers, 9: 153-179.

Bradley, R.S. \& Jones, P.D. (1992). Climate since A.D. 1500. Routledge Editorial, London, 679 pp.

Bull, W.B. (1991). Geomorphic Responses to Climatic Change. Oxford University Press, New York, 352 pp.

Chorley, R.J.; Schumm, S.A. \& Sudgen, D.E. (1984). Geomorphology. Methuen, London, 605 pp.

Constante, A.; Peña-Monné, J.L. \& Muñoz, A. (2010). Alluvial geoarchaeology of an ephemeral stream: Implications for Holocene landscape change in the central part of the Ebro depression, Northeast Spain. Geoarchaeology, 25: 475-496. http://dx.doi.org/10.1002/ gea.20314

Curry, B.B. (1999). An environmental tolerance index for ostracodes as indicators of physical and chemical factors in aquatic habitats. Palaeogeography, Palaeoclimatology, Palaeoecology, 148: 51-63. http://dx. doi.org/10.1016/S0031-0182(98)00175-8

De Deckker, P. \& Forester, R.M. (1988). The use of ostracods to reconstruct continental palaeoenvironmental records. In: Ostracoda in the Earth Sciences (De Deccker, P.; Colin, J.P. \& Peypouquet, J.P., Eds.). Elsevier, Amsterdam, 175-199.

Diebel, K. \& Piertrzeniuk, E. (1990). Pleistocene ostracods from Vértesszölös. In: Vértesszölös: site, man and culture (Kretzoi, M. \& Dobosi, V.T., Eds.). Akadémiai Kiadó, Budapest, 145-161.
Eybergen, F.A. \& Imeson, A.C. (1989). Geomorphologic processes and climatic change. Catena, 16: 307-319. http://dx.doi.org/10.1016/0341-8162(89)90017-9

Faust, D.; Zielhofer, C.; Baena, R. \& Díaz del Olmo, F. (2004). High-resolution fluvial record of late Holocene geomorphic change in northern Tunisia: climatic or human impact? Quaternary Science Reviews, 23: 1757-1775. http://dx.doi.org/10.1016/j.quascirev.2004.02.007

Fuhrmann, R. (2012). Atlas quartärer und rezenter Ostrakoden Mitteldeutschlands. Natur. Mus. Mauritianum, Thuringen. Altenburger Naturwissenschaftliche Forschungen, 15, $320 \mathrm{pp}$.

Gascón, S.; Boix, D. \& Sala, J. (2009). Are different biodiversity metrics related to the same factors? A case study from Mediterranean wetlands. Biological Conservation, 142: 2602-2612. http://dx.doi.org/ 10.1016/j.biocon.2009.06.008

Gómez-Paccard, M.; Larrasoaña, J.C.; Sancho, C.; Muñoz, A.; McDonald, E.; Rhodes, E.J.; Osácar, M.C.; Costa, E. \& Beamud, E. (2013). Environmental response of a fragile, semiarid landscape (Bardenas Reales Natural Park, NE Spain) to Early Holocene climate variability:A paleo- and environmental-magnetic approach. Catena, 103: 30-43. http://dx.doi.org/10.1016/j.catena.2011. 05.013

González, M.A. (2001). Recent formation of arroyos in the Little Missouri Badlands of southwestern North Dakota. Geomorphology, 38: 63-84. http://dx.doi. org/10.1016/S0169-555X(00)00070-2

González-Sampériz, P.; Utrilla, P.; Mazo, C.; ValeroGarcés, B.; Sopena, M.C.; Morellón, M.; Sebastián, M.; Moreno, A. \& Martínez-Bea, M. (2009). Patterns of human occupation during the early Holocene in the Central Ebro Basin (NE Spain) in response to the $8.2 \mathrm{ka}$ climatic event. Quaternary Research, 71: 121-132. http://dx.doi.org/10.1016/j.yqres.2008.10.006

Griffiths, H.I. \& Butlin, R.K. (1994). Darwinula stevensoni: a brief review of the biology of a persistent parthenogen. In: The Evolutionary Ecology of reproductive Modes in Non-Marine Ostracoda (Horne, D.H. \& Martens, K., Eds.). Greenwich University Press, 27-36.

Griffiths, H.I. \& Holmes, J.A. (2000). Non-marine ostracods and Quaternary palaeoenvironments. Technical Guide 8, Quaternary Research Association, London, $188 \mathrm{pp}$.

Hartman, G. \& Puri, H.S. (1974). Summary of Neontological and Paleontological classification of Ostracoda. Mitteilungenaus dem Hamburgischen Zoologischen Museum und Institut, 70: 7-73.

Henderson, P.A. (1990). Fresh water Ostracoda: keys and notes for the identification of the species. In: Synopses of the British fauna (new series) Number 42 (Kernack, D.M. \& Barnes, R.S.K., Eds.). Linnean Society of London, London, $228 \mathrm{pp}$.

Horne, D.J.; Cohen, A. \& Martens, K. (2002). Taxonomy, morphology and biology of Quaternary and 
living ostracoda. In: The Ostracoda. Applications in Quaternary Research (Holmes, J. \& Chivas, A.R., Eds.). American Geophysical Union, Washington, 5-36. http://dx.doi.org/10.1029/131GM02

Horne, D.J.; Curry, B.B. \& Mesquita-Joanes, F. (2012). Mutual climatic range methods for Quaternary ostracods. In: Ostracoda as Proxies for Quaternary Climate Change (Horne, D.J.; Holmes, J.; Rodríguez-Lázaro, J. \& Viehberg, F., Eds.). Developments in Quaternary Science, 17, Elsevier, 65-84. http://dx.doi.org/10.1016/ B978-0-444-53636-5.00005-6

Iriarte, M.J. \& Meaza, G. (1996). Las Bardenas Reales: aproximación a la evolución del paisaje vegetal desde mediados del segundo milenio A.C. a la actualidad. In: Biogeografía Pleistocena-Holocena de la Península Ibérica (Ramil-Rego, P.; Fernández-Rodríguez, C. \& Rodríguez Gutián, M., Eds.). Universidade de Santiago de Compostela, A Coruña, 137-148.

Janz, H. (1994). Zur Bedeutung des Schalenmerkrnals "Marginalrippen" der Gattung Ilyocypris (Ostracoda, Crustacea). Stuttgarter Beitdige zur Naturkunde Ser. B, 206: 1-19.

Karanovic, I. \& Lee, W. (2013). On the ostracod genus Ilyocypris, with description of one new species from Korea and the first report of males of I. bradyi (Crustacea: Ostracoda: Podocopida). Proceedings of the Biological Society of Washington, 126: 39-71. http://dx.doi.org/10.2988/0006-324X-126.1.39

Kempf, E.K. (1980-1997). Index and bibliography of nonmarine Ostracoda. Geologisches Institut der Universität zu Köln, 35: 188 pp.; 36: 180 pp.; 37: 204 pp.; 38: 186 pp.; 77: 232 pp.; 109: 142 pp.; 110: 134 pp.; 111: 152 pp.; 112: 144 pp.

Knox, J.C. (1984). Fluvial responses to small scale climate changes. In: Developments and Applications of Geomorphology (Costa, J.E. \& Fleischer, P.J., Eds.). Springer Verlag, Berlin, 318-342. http://dx.doi.org/ 10.1007/978-3-642-69759-3_10

Külköylüoğlu, O. (1999). Taxonomy, ecology and biogeographic distribution of spring water Ostracoda (Crustacea) in Nevada. Ph.D. thesis, University of Nevada, 228 pp.

Lamb, H.H. (1982). Climate, History and the Modern World. Methuen, London, 387 pp.

Larrasoaña, J.C.; Murelaga, X. \& Garcés, M. (2006). Magnetobiochronology of Lower Miocene (Ramblian) continental sediments from the Tudela Formation (western Ebro basin, Spain). Earth and Planetary Science Letters, 243: 409-423. http://dx.doi.org/ 10.1016/j.eps1.2006.01.034

Margalef, R. (1953). Los crustáceos de las aguas continentales ibéricas. Instituto Forestal de Investigaciones y Experiencias, Madrid, 243 pp.

Martens, K. (1992). A reassessment of Paralimnocythere Carbonnel, 1965 (Crustacea, Ostracoda, Limnocytherinae), with a description of a new genus and two new species. Bulletin van het Koninklijk Belgisch
Institut voor Natuurwetenschappen, Biologie, 62: 125-158.

Martínez-García, B.; Suarez-Hernando, O.; Suárez-Bilbao, A.; Pascual, A.; Ordiales, A.; Larrasoaña, J.C.; Murelaga, X. \& Ruiz-Sánchez, F.J. (2014). Asociaciones de ostrácodos del Mioceno tempranomedio de Loma Negra (Bardenas Reales de Navarra, Cuenca del Ebro): evolución paleoambiental de un medio lacustre. Ameghiniana, 51: 405-419. http:// dx.doi.org/10.5710/AMGH.14.08.2014.2745

Mazzini, I.; Gliozzi, E.; Rossetti, G. \& Pieri, V. (2014). The Ilyocypris puzzle: A multidisciplinary approach to the study of phenotypic variability. International Review of Hydrobiology, 99: 395-408. http://dx.doi. org/10.1002/iroh.201301729

McGregor, D.L. (1969). The reproductive potential, life history and parasitisms of the freshwater ostracod Darwinula stevensoni. In: The taxonomy, morphology and ecology of recent Ostracoda (Neale, J.W., Ed.). Oliver \& Boyd, Edinburgh, 194-221.

Meisch, C. (2000). Freshwater Ostracoda of Western and Central Europe. Spektrum Akademischer, Verlag, Heidelberg, 522 pp.

Mezquita, F.; Sanz-Brau, A. \& Miracle, M.R. (1996). New data on freshwater ostracod assemblages (Crustacea, Ostracoda) from Cuenca (Central Spain). Bulletin de la Société des Naturalistes Luxembourgeois, 97: 239-247.

Mezquita, F.; Tapia, G. \& Roca, J.R. (1999). Ostracoda from springs on the eastern Iberian Peninsula: ecology, biogeography and palaeolimnological implications. Palaeogeography, Palaeoclimatology, Palaeoecology, 148: 65-85. http://dx.doi.org/10.1016/S0031-0182(98) 00176-X

Mezquita, F.; Roca, J.R.; Reed, J.M. \& Wansard, G. (2005). Quantifying species-environment for ecological and palaeoecological studies: Examples using Iberian data. Palaeogeography, Palaeoclimatology, Palaeoecology, 225: 93-117. http://dx.doi.org/10.1016/j.palaeo. 2004.02.052

Morellón, M.; Valero-Garcés, B.; Vegas-Vilarrúbia, T.; González-Sampériz, P.; Romero, O.; Delgado-Huertas, A.; Mata, P.; Moreno, A.; Rico, M. \& Corella, J.P. (2009). Lateglacial and Holocene palaeohydrology in the western Mediterranean regions: La Lake Estanya record (NE Spain). Quaternary Science Reviews, 28: 2582-2599. http://dx.doi.org/10.1016/j.quascirev. 2009.05.014

Moreno, A.; Pérez, A.; Frigola, J.; Nieto-Moreno, V.; Rodrigo-Gámiz, M.; Martrat, B.; GonzálezSampériz, P.; Morellón, M.; Martín-Puertas, C.; Corella, J.P.; Belmonte, A.; Sancho, C.; Cacho, I.; Herrera, G.; Canals, M.; Grimalt, J.O.; JiménezEspejo, F.; Martínez-Ruiz, F.; Vegas-Vilarrúbia, T. \& Valero-Garcés, V.L. (2012). The Medieval Climate Anomaly in the Iberian Peninsula reconstructed from marine and lake records. Quaternary Science 
Reviews, 43: 16-32. http://dx.doi.org/10.1016/j. quascirev.2012.04.007

Murelaga, X.; Larraz, M.; Sancho, C.; Muñoz, A. \& Ortega, L.A. (2008). Gasterópodos del registro aluvial holoceno en Bardenas Reales de Navarra. Geogaceta, 44: 127-130.

Murelaga, X.; Ortega, L.A.; Sancho, C.; Muñoz, A.; Osácar, C. \& Larraz, M. (2012). Succession and stable isotope composition of gastropods in Holocene semiarid alluvial sequences (Bardenas Reales, Ebro Basin, NE Spain): Palaeoenvironmental implications. The Holocene, 22: 1047-1060. http://dx.doi.org/10.1177/ 0959683612437869

Niinemets, E. (1999). Ostracods. In: Lake Peipsi. Geology (Miidel, A. \& Raukas, A., Eds.). Sulemees Publishers, Tallinn, 90-97.

Ortega, L.A.; Murelaga, X.; Sancho, C.; Muñoz, A.; Osácar, C. \& Larraz, M. (2009). Composición isotópica de gasterópodos en secuencias aluviales holocenas de Bardenas Reales (Navarra): Implicaciones paleoambientales. Geogaceta, 46: 139-142.

Peña, J.L.; Echeverría, M.T.; Chueca, J. \& Julián, A. (2000). Processus géomorphologiques d'accumulation et incision pendant l'Antiquité Classique et ses rapport avec l'activité humaine et les changements climatiques holocènes dans la vallée de la Huerva (Bassin de 1'Ebre, Espagne). In: Geoarchaeology of the Landscapes of Classical Antiquity (Vermeulen, F. \& de Dapper, M., Eds.). Peeters, Leuven, 151-159.

Peña, J.L.; Julián, A.; Chueca, J.; Echeverría, M.T. \& Ángeles, G.R. (2004). Etapas de evolución holocena en el valle del río Huerva: Geomorfología y Geoarqueología. In: Geografía Física de Aragón: aspectos generales y temáticos (Peña J.L.; Longares, L.A. \& Sánchez, M., Eds.). Universidad de ZaragozaInstitución Fernando el Católico, Zaragoza, 289-302.

Poquet, J.M. \& Mesquita-Joanes, F. (2011). Combined effects of local environment and continental biogeography on the distribution of Ostracoda. Freshwater Biology, 56: 448-469. http://dx.doi.org/10.1111/ j.1365-2427.2010.02511.x

Ranta, E. (1979). Population biology of Darwinula stevensoni (Crustacea, Ostracoda) in an oligotrophic lake. Annales Zoologici Fennici, 16: 28-35.

Reed, J.M.; Mesquita-Joanes, F. \& Griffiths, H.I. (2012). Multi-indicator conductivity transfer functions for Quaternary palaeoclimate reconstruction. Journal of
Palaeolimnology, 47: 251-275. http://dx.doi.org/ 10.1007/s10933-011-9574-1

Roca, J.R. \& Baltanás, A. (1993). Ecology and distribution of ostracoda in Pyrenean springs. Journal of Crustacean Biology, 13: 165-174.

Rodríguez-Lázaro, J. \& Ruiz Muñoz, F. (2012). A general introduction to ostracods: morphology, distribution, fossil record and applications. In: Ostracoda as Proxies for Quaternary Climate Change (Horne, D.J.; Holmes, J.; Rodríguez-Lázaro, J. \& Viehberg, F., Eds.). Developments in Quaternary Science, 17, Elsevier, 1-14. http:// dx.doi.org/10.1016/B978-0-444-53636-5.00001-9

Sancho, C.; Peña, J.L.; Muñoz, A.; Benito, G.; McDonald, E.; Rhodes, E.J. \& Longares, L.A. (2008). Holocene alluvial morphopedosedimentary record and environmental changes in the Bardenas Reales Natural Park (NE Spain). Catena, 73: 225-238. http://dx.doi. org/10.1016/j.catena.2007.09.011

Saz, M.A. (2003). Temperaturas y precipitaciones en la mitad norte de España desde el siglo XV: Estudio dendroclimático. Consejo de Protección de la Naturaleza de Aragón, Zaragoza, 293 pp.

Thorndycraft, V.R. \& Benito, G. (2006). The Holocene fluvial chronology of Spain: Evidence from a newly compiled radiocarbon database. Quaternary Science Reviews, 25: 223-234. http://dx.doi.org/10.1016/j. quascirev.2005.07.003

Trudgill, S.T. (1976). Rock weathering and climate: quantitative and experimental aspects. In: Geomorphology and Climate (Derbyshire, E., Ed.). John Willey and Sons, London, 55-99.

Valero-Garcés, B.L.; Moreno, A.; Navas, A.; Mata, P.; Machín, J.; Delgado-Huertas, A.; González-Sampériz, P.; Schwalb, A.; Morellón, M.; Cheng, H. \& Edwards, R.L. (2008). The Taravilla lake and tufa deposits (Central Iberian Range, Spain) as palaeohydrological and palaeoclimatic indicators. Palaeogeography, Palaeoclimatology, Palaeoecology, 259: 136-156. http://dx.doi.org/10.1016/j.palaeo.2007.10.004

Viles, H.A. \& Goudie, A.S. (2003). Interannual, decadal and multidecadal scale climatic variability and geomorphology. Earth-Science Reviews, 61: 105-131. http://dx.doi.org/10.1016/S0012-8252(02)00113-7

Wu, Y.H.; Wu, R.J.; Wang, Q.; Zhu, Y.X. \& Pan, H.X. (2001). Palaeoclimatic variation and lake level fluctuation in Yuncheng Basin, Shanxi Province since $11 \mathrm{Ka}$ BP. Marine Geology and Quaternary Geology, 21: 83-86. 